\title{
No Tactile Prompts/Feedback
}

National Cancer Institute

\section{Source}

National Cancer Institute. No Tactile Prompts/Feedback. NCI Thesaurus. Code C133559.

Problem associated with the device ceasing to provide tactile feedback. 